RECENT DEVELOPMENTS

\author{
IN FUNCTIONAL EQUATIONS AND INEQUALITIES \\ BANACH CENTER PUBLICATIONS, VOLUME 99 \\ INSTITUTE OF MATHEMATICS \\ POLISH ACADEMY OF SCIENCES \\ WARSZAWA 2013
}

\title{
CONVEXITY AND ALMOST CONVEXITY IN GROUPS
}

\author{
WITOLD JARCZYK \\ Faculty of Mathematics, Computer Science and Econometrics, University of Zielona Góra \\ Szafrana 4a, PL-65-516 Zielona Góra, Poland \\ E-mail:w.jarczyk@wmie.uz.zgora.pl
}

\begin{abstract}
We give a review of results proved and published mostly in recent years, concerning real-valued convex functions as well as almost convex functions defined on a (not necessarily convex) subset of a group. Analogues of such classical results as the theorems of Jensen, Bernstein-Doetsch, Blumberg-Sierpiński, Ostrowski, and Mehdi are presented. A version of the Hahn-Banach theorem with a convex control function is proved, too. We also study some questions specific for the group setting, for instance the problem of the extendibility of a convex function from a subgroup to the whole group. What concerns almost convexity we present an abstract version of Kuczma's theorem. We sketch also some possible applications in improving regularity of solutions of a difference equation and in integer programming. The first appears, among others, in probability while determining weak generalized stable distributions, whereas the second is important in economics.
\end{abstract}

Introduction. Our starting point is the paper 18 by Jensen where a real function $f$ defined on an interval $I$ of reals is said to be convex if

$$
2 f(x) \leq f(x+h)+f(x-h)
$$

holds for all $x, h \in \mathbb{R}$ such that $x, x+h, x-h \in I$ or, equivalently,

$$
f\left(\frac{x+y}{2}\right) \leq \frac{f(x)+f(y)}{2}
$$

for all $x, y \in I$. These functions are also called J-convex or midconvex in the literature. A comprehensive theory of convex functions defined on a convex subset of a linear space $X$ over the rationals, mostly of a real normed space, can be found in the monograph 32 by Roberts and Varberg; for the case $X=\mathbb{R}^{n}$ the reader is referred also to the book [22]

2010 Mathematics Subject Classification: Primary 26 02, 39 02, 26A51, 39B62; Secondary 22A10, 39A40, 39A06, 90C10.

Key words and phrases: Convex function, almost convex function, Abelian group, locally compact group, extendibility of a function, set $\sigma$-ideal, difference equation, regularity of solution, integer programming, discrete convexity.

The paper is in final form and no version of it will be published elsewhere. 
by Kuczma. While extending the notion of convexity to a group setting it is clear that conditions 0.1 and $(0.2)$ are no longer equivalent: the second one demands that the group $X$ is (uniquely) divisible by 2 . There is also another advantage of condition (0.1) held for all $x, h \in \mathbb{R}$ with $x, x+h, x-h$ lying in the domain $A$ of $f$ : it imposes no additional assumption on $A$ like "convexity".

In what follows, given a subset $A$ of a group $G$, we say that $f: A \rightarrow \mathbb{R}$ is convex if 0.1 holds for all $x, h \in G$ such that $x, x+h, x-h \in A$.

The aim of the paper is to present some recent results on convex functions defined on subsets of groups. In general we are not interested here in cases of groups with an additional structure, among others in general linear spaces, Lie groups, and groups of matrices.

The contents of the article reads as follows:

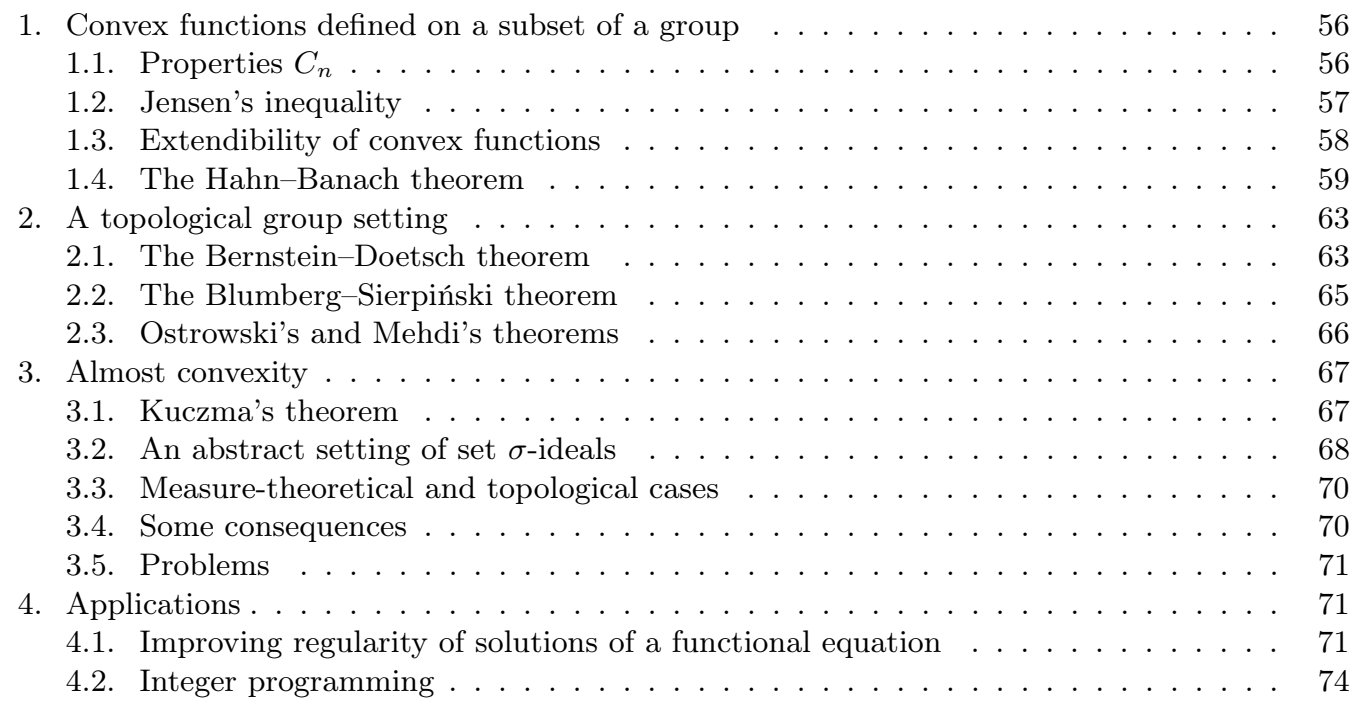

At the end of each of Sections 1-3 we pose some open problems. We omit proofs as most of the presented results have been already published. The only exceptions are the proofs of unpublished results of Subsection 1.4 devoted to Hahn-Banach theorem as well as that of Theorem 4.2.

1. Convex functions defined on a subset of a group. Most of the results of this section, mainly those presented in Subsections 1.1-1.3, come from the paper [15]. In general we require no topological structure of the group. However, in the whole section we assume that the group is Abelian.

1.1. Properties $\boldsymbol{C}_{\boldsymbol{n}}$. We begin with a discussion of the following variants of the notion of convexity.

Let $A$ be a subset of an Abelian group and $f: A \rightarrow \mathbb{R}$. We say that $f$ has property $C_{n}$ if

$$
n f(x) \leq f\left(x_{1}\right)+\ldots+f\left(x_{n}\right)
$$


whenever $x, x_{1}, \ldots, x_{n} \in A$ and $n x=x_{1}+\ldots+x_{n}$. Thus $C_{1}$ means nothing and $C_{2}$ is equivalent to the convexity of $f$. Clearly $C_{n+1}$ implies $C_{n}$ for every $n \in \mathbb{N}$. However, as follows from the examples below, the converse fails to be true.

Example 1.1 ([15, Ex.1.7]). Consider the circle group $\mathbb{R} / \mathbb{Z}$, i.e. $[0,1)$ with the addition modulo 1. Let $A=[0,1 / 3]$ and define $f: A \rightarrow \mathbb{R}$ by $f(x)=x$. Then $f$ is convex. Taking $a=1 / 3$ we have $3 a=0=0+0+0$ and $3 f(a)=1>f(0)+f(0)+f(0)=0$. Consequently, $f$ does not satisfy $C_{3}$.

Example 1.2 ([15, Ex.1.8]). It is easy to verify that any function $f:\{(0,0),(1,1)$, $(-1,0),(0,-1)\} \rightarrow \mathbb{R}$ can be extended to $\mathbb{Z}^{2}$ as a convex function (for details see [15]). Taking $f$ such that $f(0,0)=1$ and $f(1,1)=f(-1,0)=f(0,-1)=0$ we come to a function that violates $C_{3}$, as $3(0,0)=(1,1)+(-1,0)+(0,-1)$.

It turns out that under two additional conditions imposed on the domain $A$ all the properties $C_{n}, n \geq 2$, are equivalent, so all of them describe, in fact, convexity. The first of these additional assumptions is the equality $A+A=2 A$. The second one is the convexity of $A$ meant as follows.

A subset $A$ of an Abelian group $G$ is said to be convex if $x+h, x-h \in A$ implies $x \in A$ for every $x, h \in G$. Note that if $G$ is divisible by 2 and $A \subset G$ is convex, then $A+A=2 A$. If $G$ is uniquely divisible by 2 , then the convexity of $A$ is equivalent to $A+A=2 A$. In general the condition of convexity of the set $A$ is independent on the condition $A+A=2 A$. To see this observe first of all that every Abelian group is convex, but not necessarily such a group $G$ satisfies $G+G=2 G$, as not every group is divisible by 2 . On the other hand for the set $A=[0,1 / 3]$ considered in Example 1.1 we have $A+A=2 A$ but $A$ is not convex, as $2 / 3+1 / 3 \in A, 2 / 3-1 / 3 \in A$, and $2 / 3 \notin A$.

The result announced above reads as follows.

TheOREm 1.3 ([15, Th. 1.5]). Let $A$ be a convex subset of an Abelian group $G$ such that $A+A=2 A$. A function $f: A \rightarrow \mathbb{R}$ is convex if and only if it satisfies $C_{n}$ for every integer $n \geq 2$.

Corollary 1.4 ([15, Cor. 1.6]). Let $A$ be a convex subset of an Abelian group divisible by 2. A function $f: A \rightarrow \mathbb{R}$ is convex if and only if it satisfies $C_{n}$ for every integer $n \geq 2$.

1.2. Jensen's inequality. Studying convex functions in the classical setting one comes to a very fundamental inequality

$$
f(\lambda x+(1-\lambda) y) \leq \lambda f(x)+(1-\lambda) f(y) .
$$

More precisely: if $f$ is a convex function defined on a $\mathbb{Q}$-convex subset $A$ of a linear space over the rationals, then it satisfies (1.1) for every $x, y \in A$ and $\lambda \in \mathbb{Q} \cap[0,1]$ (see [32, Chap. VII, Sec. 71, Th. A]). It turns out that (1.1) holds in every Abelian group $G$ for every $\lambda \in \mathbb{Q} \cap[0,1]$ whenever $\lambda x+(1-\lambda) y$ can be interpreted in $G$. To specify the last phrase we introduce the following notation.

Let $G$ be an Abelian group. Given $x, y \in G$ and $\lambda \in \mathbb{Q}$ we write $u \sim \lambda x+(1-\lambda) y$ with some $u \in G$, if there are coprime integers $k$ and $n \neq 0$ such that $\lambda=k / n$ and $n u=k x+(n-k) y$. In general neither the existence nor the uniqueness of such an 
element $u \in G$ can be claimed. We have the following result yielding a counterpart of inequality 1.1 in a group setting.

Theorem 1.5 ([15, Th. 1.1 and Cor. 1.9]). Let $A$ be a subset of an Abelian group $G$. Assume that either $A=G$, or $A$ is convex and $A+A=2 A$. If $f: A \rightarrow \mathbb{R}$ is a convex function, then

$$
f(u) \leq \lambda f(x)+(1-\lambda) f(y)
$$

holds for every $x, y, u \in A$ and $\lambda \in \mathbb{Q} \cap[0,1]$ such that $u \sim \lambda x+(1-\lambda) y$.

Sometimes, for instance when the group $G$ is torsion free, we can claim the uniqueness of elements $u$ satisfying $u \sim \lambda x+(1-\lambda) y$. Then, if $x, y \in G, \lambda \in \mathbb{Q}$ and there is such a $u \in A \subset G$, we say that $\lambda x+(1-\lambda) y$ exists in $A$ and write $\lambda x+(1-\lambda) y=u$. The next result is an immediate consequence of the previous one.

TheOrem 1.6. Let $A$ be a subset of a torsion free Abelian group $G$. Assume that either $A=G$, or $A$ is convex and $A+A=2 A$. If $f: A \rightarrow \mathbb{R}$ is a convex function, then inequality 1.1 holds for every $x, y \in A$ and $\lambda \in \mathbb{Q} \cap[0,1]$ such that $\lambda x+(1-\lambda) y$ exists in $A$.

We complete this subsection with two corollaries concerning groups with a richer, viz. linear, structure.

Corollary 1.7 ([15, Cor. 1.10]). Let $A$ be a convex subset of a linear space over the rationals, and let $f: A \rightarrow \mathbb{R}$ be a convex function. Then inequality (1.1) holds for every $x, y \in A$ and $\lambda \in \mathbb{Q} \cap[0,1]$ such that $\lambda x+(1-\lambda) y \in A$.

This result slightly generalizes that classical one cited at the very beginning of the present subsection. There the condition of $\mathbb{Q}$-convexity of the domain $A$ means that

$$
\lambda x+(1-\lambda) y \in A
$$

whenever $x, y \in A$ and $\lambda \in \mathbb{Q} \cap[0,1]$, whereas the convexity of $A$ assumed in Corollary 1.7 (and in Corollary 1.8 also!) requires the above relation to be held for all $x, y \in A$ and for every dyadic $\lambda \in[0,1]$ only.

Corollary 1.8 ([15, Cor. 1.11]). Let $A$ be a convex subset of a linear topological space and let $f: A \rightarrow \mathbb{R}$ be a continuous convex function. Then inequality (1.1) holds for every $x, y \in A$ and $\lambda \in[0,1]$ such that $\lambda x+(1-\lambda) y \in A$.

1.3. Extendibility of convex functions. This topic is not very typical in the classical theory. However, in the group setting the problem has surprisingly turned out to be not trivial. We start with a simple observation which is another immediate consequence of Theorem 1.3 .

Corollary 1.9 ([15, Cor. 2.1]). Let $A$ be a convex subset of an Abelian group $G$ such that $A+A=2 A$. If a real function $f$, defined on a subset of $A$, has a convex extension to $A$, then $f$ satisfies the condition $C_{n}$ for every $n \in \mathbb{N}$.

Of course, any convex function $f: A \rightarrow \mathbb{R}$ is trivially extendible to $A$ as a convex function, so Examples 1.1 and 1.2 show that each of the assumptions imposed on the set $A$ is essential in Corollary 1.9 . 
Denote by $H$ the group generated by two real numbers linearly independent over the rationals. Then $H$ is isomorphic to the group $\mathbb{Z}^{2}$. Making use of the function described in Example 1.2 we can obtain a convex function $f: H \rightarrow \mathbb{R}$ which does not satisfy $C_{3}$, and thus, by Corollary 1.9 , cannot be extended to $\mathbb{R}$ as a convex function. Consequently, we have come to the following result.

THEOREM 1.10 ([15, Th. 2.2]). There exists a convex function defined on a subgroup of the reals which cannot be extended to $\mathbb{R}$ as a convex function.

Rather unexpectedly it turns out that the property of satisfying all the conditions $C_{n}$ is much more appropriate while extending the function.

TheOREM 1.11 ([15, Th. 2.3]). Let $H$ be a subgroup of an Abelian group $G$. If $f: H \rightarrow \mathbb{R}$ satisfies $C_{n}$ for every $n \in \mathbb{N}$, then $f$ can be extended to $G$ as a function satisfying $C_{n}$ for every $n \in \mathbb{N}$.

The proof of this theorem (see [15]) is fairly non-trivial. Given any $x \in G \backslash H$ the required extension to the group $\langle H, x\rangle$ generated by $H$ and $x$ is defined step by step. Among the tools used in the proof there is the fundamental theorem of finitely generated Abelian groups (cf. [34, Th. 10.26]). The final step is making use of Zorn's lemma.

The assumption that $H$ is a subgroup of $G$ is essential for the validity of Theorem 1.11, which can be seen from the following simple example. Let $A=(a, b)$, where $-\infty<a<b<\infty$, and let $f: A \rightarrow \mathbb{R}$ be a convex function such that $f(a+)=f(b-)=\infty$. By Theorem 1.3. the function $f$ satisfies $C_{n}$ for every $n \in \mathbb{N}$. On the other hand it does not admit a convex extension to $\mathbb{R}$.

As an immediate consequence of Theorems 1.3 and 1.11 we obtain the following result. Corollary 1.12 ([15, Cor. 2.4]). Let $H$ be a subgroup of an Abelian group $G$. If $H$ is divisible by 2 , then every convex function defined on $H$ can be extended to $G$ as a convex function.

Comparing Theorem 1.10 and Corollary 1.12 we see how subtle the situation is while extending functions from a subgroup $H$ to the whole group, with preserving convexity. The crucial role is played here by the assumption of divisibility by 2 of the subgroup $H$.

For a little bit relative problem of extendibility of convex functions defined on a finite set of reals to the whole real line the reader is referred to the paper [29] by Pinkus and Wulbert. Actually that paper deals with the notion of $n$-convexity introduced already by Eberhard Hopf in his dissertation [12, and then extensively studied by Popoviciu in his thesis [30, and his monograph [31. In the reals that notion generalizes the convexity considered here. The paper [29] brings a (nonconstructive) method of determining if an $n$-convex function defined on a finite set of reals has a desired $n$-convex extension to $\mathbb{R}$.

1.4. The Hahn-Banach theorem. A version of that classical result, formulated as Theorem 1.15 below, was not published yet, so here it is presented with a proof. The main ideas are due to Miklós Laczkovich (private communication).

Let $S$ be a semigroup. A function $p: S \rightarrow \mathbb{R}$ is said to be subadditive if $p(x+y) \leq$ $p(x)+p(y)$ for every $x, y \in S$. If $X$ is a linear space over the reals then $p: X \rightarrow \mathbb{R}$ is 
called sublinear if it is subadditive and $p(t x)=t p(x)$ for every $x \in X$ and nonnegative $t \in \mathbb{R}$. The Hahn-Banach theorem states that

(HB) if $X$ is a linear space over the reals, $p: X \rightarrow \mathbb{R}$ is sublinear, $Y$ is a subspace of $X$, and $L: Y \rightarrow \mathbb{R}$ is a linear map such that $L(x) \leq p(x)$ for every $x \in Y$, then $L$ can be extended to $X$ as a linear map such that $L(x) \leq p(x)$ for every $x \in X$.

Consider the following variants of (HB).

(HB1) Let $X$ be a linear space over the rationals and let $p: X \rightarrow \mathbb{R}$ be subadditive. If $Y$ is a subspace of $X$, and $a: Y \rightarrow \mathbb{R}$ is an additive map such that $a(x) \leq p(x)$ for every $x \in Y$, then a can be extended to $X$ as an additive map such that $a(x) \leq p(x)$ for every $x \in X$.

(HB2) Let $X$ be a linear space over the rationals and let $p: X \rightarrow \mathbb{R}$ be convex. If $Y$ is a subspace of $X$, and $a: Y \rightarrow \mathbb{R}$ is an additive map such that $a(x) \leq p(x)$ for every $x \in Y$, then a can be extended to $X$ as an additive map such that $a(x) \leq p(x)$ for every $x \in X$.

It is easy to see that each of (HB1) and (HB2) implies (HB). Indeed, if $p$ is sublinear then it is both subadditive and convex. Also, if $L \leq p$ is additive then $L$ is linear. To prove this, let $x \in X$ be given. Then the map $\mathbb{R} \ni t \mapsto L(t x)$ is additive, and bounded from above on an interval. As is well-known (cf., for instance, 22, Th. 5.2.1] and the comment just after it), this implies that $L(t x)=t L(x)$ for every $t \in \mathbb{R}$, proving that $L$ is linear.

The statements (HB1) and (HB2) are independent in that neither of them is covered by the other. For example, if $X=\mathbb{R}$ then $p: X \rightarrow \mathbb{R}$, given by $p(x)=\sqrt{|x|}$, is subadditive but not convex, while $p(x)=x^{2}$ defines a function $p: X \rightarrow \mathbb{R}$ which is convex but not subadditive.

It was proved by R. Kaufman in [19] and 20, that (HB1) can be generalized to Abelian groups, and even to Abelian semigroups; see also [33] for a generalization.

In this section we shall consider the possible generalization of (HB2) to Abelian groups. We shall prove that (HB2) holds under the following conditions: $p$ has the property $C_{n}$ for every $n \in \mathbb{N}$, and $Y$ is divisible by 2 . As the following examples show, none of these conditions can be omitted.

Example 1.13. Let $G=\mathbb{Q}$ and define $p: G \rightarrow \mathbb{R}$ by $p(x)=x^{2}-x$. Then $p$ is convex and, in addition, has property $C_{n}$ for every $n \in \mathbb{N}$. Take $H=\mathbb{Z}$, and let $a$ be the zero function on $H$. Then $a \leq\left. p\right|_{H}$. Now the only extension of $a$ to $\mathbb{Q}$ as an additive function is the identically zero function which does not satisfy $a \leq p$.

EXAMPLE 1.14. In Example 1.2 we described a convex function $f$ on $G=\mathbb{Z}^{2}$ such that $f(0,0)=1$ and $f(1,1)=f(-1,0)=f(0,-1)=0$. Then $p=f-1$ is convex and $p(0,0)=0$. The subgroup $H=\{(0,0)\}$ is divisible by 2 and $a \leq\left. p\right|_{H}$, where $a(0,0)=0$. However, there is no additive function $\bar{a}: \mathbb{Z}^{2} \rightarrow \mathbb{R}$ with $\bar{a} \leq p$. Indeed, suppose that $\bar{a}$ is such a homomorphism. Then each of the numbers $\bar{a}(1,1), \bar{a}(-1,0), \bar{a}(0,-1)$ is not greater than -1 . On the other hand, $\bar{a}(1,1)+\bar{a}(-1,0)+\bar{a}(0,-1)=\bar{a}(0,0)=0$, which is impossible. 
THEOREM 1.15. Let $G$ be an Abelian group, $H$ its subgroup divisible by 2, and let $p: G \rightarrow \mathbb{R}$ be a function satisfying the condition $C_{n}$ for every $n \in \mathbb{N}$. Then any additive function $a: H \rightarrow \mathbb{R}$ such that $a(x) \leq p(x)$ for every $x \in H$ can be extended to $G$ as an additive function satisfying $a(x) \leq p(x)$ for every $x \in G$.

Our method of proving Theorem 1.15 is to reduce it to (HB1). For this purpose we shall need some lemmas.

Lemma 1.16 ([15, Lemma 1.2]). Let $G$ be an Abelian group, $x, h \in G$, and let $f:\{x$, $x+h, \ldots, x+n h\} \rightarrow \mathbb{R}$ be a convex function. Then

$$
n f(x+k h) \leq(n-k) f(x)+k f(x+n h)
$$

for every $k=0, \ldots, n$.

Lemma 1.17. Let $A$ be a subset of an Abelian group $G$ and let $f: A \rightarrow \mathbb{R}$ be a convex function. If $x_{0} \in G$ is of finite order, then $f\left(x+x_{0}\right)=f(x)$ for every $x \in G$ with $x+x_{0} \mathbb{Z} \subset A$.

Proof. Since $-x_{0}$ is also of finite order, it is enough to show that $f\left(x+x_{0}\right) \leq f(x)$ for every $x \in G$ such that $x+x_{0} \mathbb{Z} \subset A$. Take any such $x$ and $n \in \mathbb{N}$ satisfying $n x_{0}=0$. Then, by Lemma 1.16, we obtain

$$
n f\left(x+x_{0}\right) \leq(n-1) f(x)+f\left(x+n x_{0}\right)=n f(x)
$$

which was to be proved.

Lemma 1.18. Let $X$ be a linear space over the rationals and let $p: X \rightarrow \mathbb{R}$ be a convex function such that $p(0) \geq 0$. Then

$$
q(x)=\inf \{n p(x / n): n \in \mathbb{N}\}
$$

defines a subadditive function $q: X \rightarrow \mathbb{R}$ satisfying $q \leq p$.

Proof. It is clear that $q \leq p$. Take any $x \in X$. By Lemma 1.16 we have

$$
\begin{aligned}
0 & \leq(n+1) p(0)=(n+1) p((-x)+n(x / n)) \\
& \leq p(-x)+n p((-x)+(n+1)(x / n))=p(-x)+n p(x / n)
\end{aligned}
$$

for every $n \in \mathbb{N}$. Thus $n p(x / n) \geq-p(-x)$ for every $n \in \mathbb{N}$, which proves that $q$ is finite. We show that $q$ is subadditive. Let $x, y \in X$ be arbitrary. Then, by the definition of $q$ and Corollary 1.7 .

$$
\begin{aligned}
q(x+y) \leq(m+n) p\left(\frac{x+y}{m+n}\right)=(m+n) p\left(\frac{m}{m+n} \frac{x}{m}+\frac{n}{m+n}\right. & \left.\frac{y}{n}\right) \\
& \leq m p\left(\frac{x}{m}\right)+n p\left(\frac{y}{n}\right)
\end{aligned}
$$

for all $m, n \in \mathbb{N}$, whence $q(x+y) \leq q(x)+q(y)$.

Lemma 1.19. Any convex function defined on $\mathbb{Q}$ is continuous.

Proof. Let $f: \mathbb{Q} \rightarrow \mathbb{R}$ be a convex function. Write

$$
h_{a, b}(t)=\frac{f(b)-f(a)}{b-a}(t-a)+f(a)
$$


for every $a, b \in \mathbb{Q}, a<b$, and for every $t \in \mathbb{Q}$. Let $c \in \mathbb{Q}$ be arbitrary, and choose rational numbers $a$ and $b$ such that $a<c<b$. Take any $t \in \mathbb{Q} \cap(c, b)$. Then $a<c<t<b$, also

$$
c=\frac{t-c}{t-a} a+\frac{c-a}{t-a} t \quad \text { and } \quad t=\frac{b-t}{b-c} c+\frac{t-c}{b-c} b,
$$

whence, by Corollary 1.7 .

$$
f(c) \leq \frac{t-c}{t-a} f(a)+\frac{c-a}{t-a} f(t) \quad \text { and } \quad f(t) \leq \frac{b-t}{b-c} f(c)+\frac{t-c}{b-c} f(b) .
$$

This results in $h_{a, c}(t) \leq f(t) \leq h_{c, b}(t)$ for every $t \in \mathbb{Q} \cap(c, b)$. Since

$$
h_{a, c}(c+)=h_{c, b}(c+)=f(c),
$$

it follows that $f(c+)=f(c)$. That is, $f$ is continuous at $c$ from the right. A similar argument shows that $f$ is continuous at $c$ from the left.

Proof of Theorem 1.15. First assume that $G$ is uniquely divisible, i.e. $G$ is a linear space over the rationals. Let $q: G \rightarrow \mathbb{R}$ be defined by $(1.2)$. Then, by Lemma 1.18, $q$ is subadditive and $q \leq p$. We prove that $a \leq\left. q\right|_{H}$.

Let $x \in H$ be fixed, and put $p_{x}(t)=p(t x)$ for every $t \in \mathbb{R}$. It is easy to check that $p_{x}$ is convex on $\mathbb{Q}$. Therefore, by Lemma $1.19, p_{x}$ is continuous. Since $H$ is divisible by 2 , we have $\frac{k}{2^{n}} x \in H$ for every $k \in \mathbb{Z}$ and $n \in \mathbb{N}$, whence,

$$
\frac{k}{2^{n}} a(x)=a\left(\frac{k}{2^{n}} x\right) \leq p\left(\frac{k}{2^{n}} x\right)=p_{x}\left(\frac{k}{2^{n}}\right) .
$$

From the continuity of $p_{x}$ it follows that $t a(x) \leq p_{x}(t)$ for every $t \in \mathbb{Q}$. In particular, $a(x) \leq n p_{x}(1 / n)=n p(x / n)$ for every $n \in \mathbb{N}$, which implies $a(x) \leq q(x)$.

Now it follows from (HB1) that $a$ can be extended to $G$ as an additive function such that $a(x) \leq q(x)$ for every $x \in G$. Since $q \leq p$, this proves the theorem in that case.

Next assume that $G$ is a divisible group. Let $G_{0}$ denote the torsion subgroup of $G$. Then $p$ is constant on each coset of $G_{0}$ by Lemma 1.17. Also $a(x)=0$ for every $x \in H \cap G_{0}$. Therefore, if we define $a(h+x)=a(h)$ for every $h \in H$ and $x \in G_{0}$, then we obtain an extension of $a$ to the subgroup $H_{0}=\left\langle H, G_{0}\right\rangle$ generated by $H$ and $G_{0}$ such that $a(x) \leq p(x)$ for every $x \in H_{0}$, and $a$ is constant on each coset of $G_{0}$ lying in $H_{0}$.

The factor group $G / G_{0}$ is divisible and torsion free, that is uniquely divisible. Let $\phi$ denote the natural homomorphism from $G$ to the factor group $G / G_{0}$. Then $\phi\left(H_{0}\right)=\phi(H)$ is divisible by 2 , since it is the homomorphic image of a group with the same property. There is a function $\bar{p}: G / G_{0} \rightarrow \mathbb{R}$ such that $p=\bar{p} \circ \phi$. Clearly, $\bar{p}$ is convex on $G / G_{0}$. Similarly, there is a homomorphism $\bar{a}: \phi\left(H_{0}\right) \rightarrow \mathbb{R}$ such that $a=\bar{a} \circ \phi$. Clearly, $\bar{a} \leq \bar{p}$ on $\phi\left(H_{0}\right)$. Since $G / G_{0}$ is uniquely divisible, there exists an extension of $\bar{a}$ to $G / G_{0}$ such that $\bar{a} \leq \bar{p}$ on $G / G_{0}$. Taking $\bar{a} \circ \phi$ we obtain an extension of $a$ satisfying the requirements.

Finally, consider the general case. It is well-known that $G$ can be embedded in a divisible group $A$ (see [34, Th. 10.23]). By Theorem 1.11, $p$ can be extended to $A$ as a convex function. By what we proved above, $a$ can be extended to $A$ as an additive function such that $a \leq p$. Then the restriction of $a$ to $G$ satisfies the requirements. 
Problem. Because of the subtraction occurring in (0.1), that inequality cannot serve as a definition of the convexity in a semigroup setting. However, the condition $C_{2}$, which is equivalent to convexity in groups, is a good candidate for such a definition. In fact, all the conditions $C_{n}$ make sense in semigroups. It seems to be reasonable to study functions defined on subsets of semigroups and having the properties $C_{n}$. How far the results of Subsections 1.1-1.4 can be generalized to (Abelian) semigroups? Is it possible to have a comprehensive theory of convex functions (in the sense proposed above) in semigroups? This concerns also the results of the next sections, where topological groups could be replaced by topological semigroups.

2. A topological group setting. In this section we deal with convex functions in groups with much more richer structure, viz. topological groups, in Subsections 2.2 and 2.3 assumed to be locally compact. The results presented here originate in two papers: [6] by Chademan and Mirzapour, and [15]. We consider possible generalizations of such classical theorems as those due to Bernstein and Doetsch, Blumberg and Sierpiński, also Ostrowski. The counterparts of these theorems proved by Chademan and Mirzapour are different from those obtained in [15]. This concerns both the assumptions and the assertions. For instance, in [6] (cf. Theorems 1 and 2 there) the authors sometimes assume that if the considered topological group is not discrete, then it has the property

$(\mathrm{CM})$ for every $x \in G$ there is a sequence $\left(y_{n}\right)_{n \in \mathbb{N}}$ of elements of $G$ such that $x=2^{n} y_{n}$ for each $n \in \mathbb{N}$ and $y_{n} \rightarrow 0$,

which is not the case in [15]. On the other hand, in [15] only Abelian groups are treated, whereas the commutativity of groups in [6] is postulated only in a result generalizing Ostrowski's theorem.

The main tool of the proofs in [6] is the following generalization of Jensen's theorem.

Theorem 2.1 ([6, Prop. 3]). Let $U$ be an open subset of a locally compact not discrete group and let $x \in U$. If a convex function $f: U \rightarrow \mathbb{R}$ satisfies the condition

$$
\limsup _{h \rightarrow 0} f(x+h)<\infty,
$$

then $f$ is continuous at $x$.

In fact, Chademan and Mirzapour admit functions from slightly larger classes than the class of convex functions. Given a topological group they consider locally convex, sequentially convex at a point and locally uniformly convex at a point functions defined on a subset of the group (see [6, Sec. 2.1]). In particular, Theorem 2.1] is considered there for functions sequentially convex at $x$. Here, however, for the uniformity of the presentation, we shall formulate all the results for convex functions only.

2.1. The Bernstein-Doetsch theorem. Its classical version states that if $D$ is a convex open subset of a normed linear space and $f: D \rightarrow \mathbb{R}$ is a convex function locally bounded from above at a point, then $f$ is continuous (see [32, Chap. VII, Sec. 71, Th. C and Chap. IV, Sec. 41, Th. C] or [22, Th. 6.4.2], also [2]).

To formulate a generalization proved by Chademan and Mirzapour we need the following notion. A subset $A$ of a group $G$ is called (right) midconvex if for every $x, y \in A$ 
there is an $h \in G$ such that $x+h \in A$ and $x+2 h=y$. In the case when $G$ is Abelian the midconvexity of $A$ is equivalent to the condition $A+A=2 A$. However, in general, both properties are independent.

Theorem 2.2 ([6, Th. 1]). Let $G$ be a topological group with property (CM) whenever $G$ is not discrete and let $D$ be a midconvex open subset of $G$. If a convex function $f: D \rightarrow \mathbb{R}$ is locally bounded from above at a point, then $f$ is continuous.

The approach presented in 15] need the commutativity of the group. On the other hand, the assertion obtained there is stronger: the local uniform continuity of $f$ is stated and, in the case when the group is metric, the local Lipschitz condition. In the theorem below another notion occurs, too. Namely, we say that a neighbourhood $U \subset G$ of 0 is absorbing if for every $x \in G$ there is a positive integer $n$ such that $x \in 2^{n} U$. The detailed formulation of the result is as follows.

Theorem 2.3 ([15, Th. 3.6]). Let $G$ be an Abelian topological [metric] group and let D be an open subset of $G$ satisfying at least one of the following conditions:

(i) $D=G$;

(ii) $D$ is connected;

(iii) $D$ is convex, and every convex neighbourhood of 0 is absorbing.

If a convex function $f: D \rightarrow \mathbb{R}$ is locally bounded from above at a point, then $f$ is locally uniformly continuous [locally Lipschitz].

Clearly, condition (CM) implies that every neighbourhood of 0 is absorbing. Consequently, for Abelian groups Theorem 2.2 can be derived from Theorem 2.3 (where it is enough to assume (iii) only). Observe also that any topological group, containing at least one absorbing neighbourhood of 0 is necessarily divisible by 2 .

Note that Theorem 2.3 generalizes the Bernstein-Doetsch theorem even in the case $G=\mathbb{R}^{n}$, as the notion of convexity of sets is more general than the classical one; for that observation it is enough to consider Theorem 2.3 with assumption (iii) only.

The following two examples show that if there are convex neighbourhoods of 0 which are not absorbing, then, in general, the assertion of Theorem 2.3 fails to be true.

ExAmple 2.4 ([15, Ex. 3.4]). Consider the group $G=\mathbb{R} \times \mathbb{Z}$ with the invariant metric $d$ given by $d\left(\left(x_{1}, n_{1}\right),\left(x_{2}, n_{2}\right)\right)=\left|x_{1}-x_{2}\right|+\left|n_{1}-n_{2}\right|$. The topology induced by $d$ is the product topology when the factors $\mathbb{R}$ and $\mathbb{Z}$ are endowed with the Euclidean topology and the discrete one, respectively.

The set $D=\mathbb{R} \times\{0,1\}$ is convex and open. Let $a: \mathbb{R} \rightarrow \mathbb{R}$ be a discontinuous additive function (cf. [22, Cor. 5.2.2] for instance). Then the function $f: D \rightarrow \mathbb{R}$, given by $f(x, n)=n a(x)$, is convex. Moreover, $f$ vanishes on the open subset $\mathbb{R} \times\{0\}$ of $D$. However, $f$ is not continuous at any point of $\mathbb{R} \times\{1\}$, as $f(\cdot, 1)=a$.

ExAmple 2.5 ([15, Ex. 3.5]). Let $d_{0}$ be the discrete metric on $\mathbb{R}$, that is $d_{0}(x, y)=1$ whenever $x, y$ are distinct reals and $d_{0}(x, x)=0$ for every $x \in \mathbb{R}$. Consider the group $G=\mathbb{R} \times \mathbb{R}$ with the metric $d$ defined by $d\left(\left(x_{1}, y_{1}\right),\left(x_{2}, y_{2}\right)\right)=\left|x_{1}-x_{2}\right|+d_{0}\left(y_{1}, y_{2}\right)$. The topology generated by $d$ is the product topology when the first $\mathbb{R}$ factor is endowed with the Euclidean topology and the second $\mathbb{R}$ factor is equipped with the discrete one. 
The set $D=\mathbb{R} \times[0,1]$ is convex and open. Take any discontinuous additive function $a: \mathbb{R} \rightarrow \mathbb{R}$ and define a function $f: D \rightarrow \mathbb{R}$ putting $f(x, y)=0$ for every $x \in \mathbb{R}, y \in[0,1)$ and $f(x, 1)=a(x)^{2}$ for every $x \in \mathbb{R}$. Then $f$ is a convex function which vanishes on the open subset $\mathbb{R} \backslash\{0\}$ of $D$ but is not continuous.

Making use of Theorem 2.3 one can prove another extension result.

Corollary 2.6 ([15, Cor. 3.8]). Let $H$ be a dense subgroup of an Abelian topological group $G$ and let $f: H \rightarrow \mathbb{R}$ be a convex function. If $f$ is locally bounded from above at a point, then $f$ can be extended to $G$ as a continuous convex function.

It turns out that if we assume the global boundedness of $f$ from above, then the assertion of Theorem 2.3 can be proved in arbitrary Abelian topological [metric] group.

TheOREm 2.7 ([15, Th. 3.1]). Let D be an open subset of an Abelian topological [metric] group and let $f: D \rightarrow \mathbb{R}$ be a convex function. If $f$ is bounded from above, then it is locally uniformly continuous [locally Lipschitz].

2.2. The Blumberg-Sierpiński theorem. A well-known theorem of Blumberg and Sierpiński (see [3] and [36, also [22, Th. 9.4.2]) states that every Lebesgue measurable convex function, defined on an open convex subset of $\mathbb{R}^{k}$, is continuous. While generalizing this result to locally compact topological groups we replace the Lebesgue measurability by the $\mathfrak{M}$-measurability, where $\mathfrak{M}$ stands for completion of the $\sigma$-field of Borel subsets of the group $G$ with respect to the (say left) Haar measure in $G$; in the sequel we shall call it measurable.

We begin with a generalization proposed by Chademan and Mirzapour.

TheOrem 2.8 ([6, Cor. 2]). Let $U$ be an open subset of a locally compact topological group. If $f: U \rightarrow \mathbb{R}$ is a measurable convex function, then $f$ is continuous.

A slightly more general version of the Blumberg-Sierpiński theorem was proved for locally uniformly convex functions (see [6, Prop. 4]). In the commutative case the assertion of Theorem 2.8 can be strengthened.

TheOREM 2.9 ([15, Th. 4.1]). Let $U$ be an open subset of a locally compact Abelian topological [metric] group. If $f: U \rightarrow \mathbb{R}$ is a measurable convex function, then $f$ is locally uniformly continuous [locally Lipschitz].

The original Blumberg-Sierpiński theorem has a topological counterpart, where the Lebesgue measurability of the function is replaced by the Baire property (see [22, Ex. 7, p. 256]). By Baire property of a real-valued function we mean here the measurability with respect to the $\sigma$-field of Baire sets, viz. sets which differ from an open set by a set of the first Baire category. The mentioned generalization to groups reads as follows.

TheOREM 2.10 ([15, Th. 4.2]). Let $U$ be an open subset of a locally compact Abelian topological [metric] group. If $f: U \rightarrow \mathbb{R}$ is a convex function with the Baire property, then $f$ is locally uniformly continuous [locally Lipschitz].

The assumption of local compactness in Theorems 2.82 .10 cannot be removed, as the following shows. 
ExAmple 2.11 ([15, Rem. 4.3]). Let $\alpha$ be an irrational number. Then the group $G=$ $\mathbb{Z}+\alpha \mathbb{Z}$, endowed with the topology generated by the Euclidean topology of $\mathbb{R}$, is an Abelian topological group. Since $G$ is countable, every function defined on $G$ is Borel, whence both measurable and with the Baire property. However, there are convex, even additive, functions on $G$ which are discontinuous. The function $f: G \rightarrow \mathbb{R}$ given by $f(n+k \alpha)=k$, serves as an example.

Nevertheless, the condition of local compactness of the group in Theorem 2.10 can be relaxed. Namely, the same proof (see [15]) shows that the assertion of Theorem 2.10 is valid in every topological Abelian group $G$ in which every nonempty open set is of the second Baire category. It is easy to see that this happens if and only if $G$ is of the second category. Indeed, if there is a nonempty open set which is of the first category, then, as its translations cover $G$, it follows that $G$ is the union of a family of open sets of the first category. By Banach's Category Theorem [28, Th. 16.1], this implies that $G$ is of the first category.

2.3. Ostrowski's and Mehdi's theorems. As was proved by Ostrowski in [27] the assumptions of Lebesgue measurability of the function $f$ in the Blumberg-Sierpiński theorem may be essentially relaxed: it is enough to require that $f$ is bounded from above on a measurable set of positive measure (see also [22, Th. 9.3.1]). Examples 2.4 and 2.5 show that if we want to generalize this result to topological groups, then we have to impose additional assumptions on the group or on the domain of $f$. It can be seen from the example below that the situation is rather sophisticated: there are discontinuous convex functions defined on the whole group and bounded above on a closed set of positive measure.

ExAmPle 2.12. Let $G$ be as in Example 2.5 and take any discontinuous additive function $a: \mathbb{R} \rightarrow \mathbb{R}$. Then $f: G \rightarrow \mathbb{R}$, given by $f(x, y)=a(x)$, is discontinuous convex (even additive) function, vanishing on the closed set $F=\{0\} \times \mathbb{R}$ of positive Haar measure. In fact, $F$ is of infinite measure. Nevertheless, the interior of $F+F$ is empty. Observe also that the group $G$ is not divisible by 2 .

While proving any version of Ostrowski's theorem a crucial point is to know that $f$ under consideration is bounded above on a set $A$ such that the interior of $A+A$ is nonempty. It is well-known that in locally compact Abelian groups this is the case when $A$ is measurable set of finite ( $\sigma$-finite) positive measure (see [11, (20.17) Cor.]). It turns out that in the proof of the generalized Ostrowski's theorem it is advisable to assume the following property which is an abstract version of Steinhaus' theorem:

(S) If $G$ is a locally compact group and $A \subset G$ is a measurable set of finite positive measure, then the interior of $A+A$ contains an element $2 x$ with some $x \in G$.

Clearly, every locally compact Abelian group divisible by 2 has property (S). Also every connected locally compact Abelian group $G$ has property (S), as for such groups $2 G$ is dense in $G$. Note, however, that in general $2 G$ is not dense in $G$. This is the case, for instance, if $G$ is a compact group not divisible by 2 ; then $G \backslash 2 G$ is a nonempty open set, disjoint with $2 G$. Examples of such a group are the Cantor group $\{0,1\}^{\mathbb{N}}$ and the group of 2-adic integers. 
In [15] one can find the following generalization of Ostrowski's theorem.

THEOREM 2.13 ([15, Th. 4.4]). Let $G$ be a locally compact Abelian topological [metric] group and let $D$ be a convex open subset of $G$ satisfying at least one of the following conditions:

(i) $D=G$ and $G$ has property $(\mathrm{S})$;

(ii) $D$ is connected;

(iii) every convex neighbourhood of 0 is absorbing.

If a convex function $f: D \rightarrow \mathbb{R}$ is bounded from above on a measurable set of finite positive measure, then $f$ is locally uniformly continuous [locally Lipschitz].

This is an extension of [6. Th. 2] where the commutativity of the group is also assumed. (It seems that there the assumption of finiteness of measure of the set, on which $f$ is bounded above, has been wrongly omitted.)

The final result of this subsection is the category counterpart of Theorem 2.13, it generalizes Mehdi's theorem [24] (see also [22, Th. 9.3.2]).

THEOREM 2.14 ([15, Th. 4.4]). Let $G$ be an Abelian topological [metric] group and let D be an open subset of $G$ satisfying at least one of conditions (i)-(iii). If a convex function $f: D \rightarrow \mathbb{R}$ is bounded from above on a set of the second category with the Baire property, then $f$ is locally uniformly continuous [locally Lipschitz].

Problem. Prove or disprove that every locally compact Abelian group has property (S).

3. Almost convexity. In the paper 21] Kuczma proved that if $D$ is a convex open subset of $\mathbb{R}^{n}$, then every function $f: D \rightarrow \mathbb{R}$ satisfying inequality $(0.2$ for almost every (with respect to the product Lebesgue measure in $\mathbb{R}^{n} \times \mathbb{R}^{n}$ ) pair $(x, y) \in D \times D$ equals a convex function a.e. (with respect to Lebesgue measure in $\mathbb{R}^{n}$ ). The results of this section extend Kuczma's theorem to some locally compact Abelian groups; they originate in the papers [16] and [14].

3.1. Kuczma's theorem. Let $A$ be a subset of a locally compact Abelian group $G$ with Haar measure $\lambda$. We shall say that a function $f: A \rightarrow \mathbb{R}$ is almost convex if inequality (0.1) holds for $\lambda^{2}$-a.e. $(x, h) \in G^{2}$ such that $x, x+h, x-h \in A$. Clearly, for $G=\mathbb{R}^{n}$ and for a convex open set $A \subset G$, this is equivalent to Kuczma's condition.

The following theorem is the main result of the paper [16].

TheOrem 3.1 ([16, Th. 1]). Let $G$ be a locally compact Abelian group divisible by 2. Then for every almost convex function $f: G \rightarrow \mathbb{R}$ there exists a unique convex function $g: G \rightarrow \mathbb{R}$ such that $f(x)=g(x)$ for $\lambda$-a.a. $x \in G$.

Kuczma's theorem is valid in any compact connected Abelian group, as every such a group is divisible. The condition of divisibility by 2 is by no means necessary. Indeed, in discrete groups every almost convex function is convex, so Kuczma's theorem is true in discrete groups. It is also valid on torsion groups, as every almost convex function defined on a torsion group is constant a.e.

The outer measure of a set $E \subset G$ is defined by $\bar{\lambda}(E)=\inf \{\lambda(U): E \subset U \subset G$, $U$ is open $\}$. Then $\bar{\lambda}$ is an extension of $\lambda$ to an outer measure on $G$. It turns out that 
Kuczma's proof is based on the fact that the following properties are satisfied in the case $G=\mathbb{R}^{n}$. Here and in the sequel $\frac{1}{n} E$ denotes the set $\{x \in G: n x \in E\}$ for every $E \subset G$ and $n \in \mathbb{N}$. Moreover, we shall make use of the transformation of $G \times G$ given by $\phi(x, y)=(x+y, x-y)$. We say that $E \subset G$ is a null set if $\bar{\lambda}(E)=0$, whereas the complement of a null set is said to be of full measure. Consider the following properties.

$\left(\mathrm{P}_{1}\right)$ If $A \subset G$ is a set of positive outer measure, then also the set $\frac{1}{2} A$ is of positive outer measure.

$\left(\mathrm{P}_{2}\right)$ If $A, B \subset G$ are sets of positive outer measure and $M, N \subset G^{2}$ are sets of full measure, then

$$
\phi^{-1}(M) \cap N \cap\left[\left(\frac{1}{2} A\right) \times\left(\frac{1}{2} B\right)\right] \neq \emptyset .
$$

The proof of Theorem 3.1 presented in [16] consists of two parts. In the first one the authors prove that Kuczma's argument works in any group with properties $\left(\mathrm{P}_{1}\right)$ and $\left(\mathrm{P}_{2}\right)$. The second one, consisting of a series of lemmas, shows that both the properties are satisfied in every group which is divisible by 2 .

3.2. An abstract setting of set $\boldsymbol{\sigma}$-ideals. In the book 22] Kuczma actually proved his theorem in a more general setting, in which the families of null sets were replaced by some abstract ideals. The paper [14] provides counterparts of that result for functions defined in groups.

Sets being elements of proper $\sigma$-ideals can be considered as small sets in abstract setting. In what follows the phrases almost everywhere and almost all are meant in the sense of given $\sigma$-ideals in the groups $G$ and $G \times G$.

To fix the terminology we recall some notions. A nonempty family $\mathcal{I}$ of subsets of a set $X$ is called a $\sigma$-ideal in $X$ if every subset of any element of $\mathcal{I}$ belongs to $\mathcal{I}$ and the union of any countable subfamily of $\mathcal{I}$ is in $\mathcal{I}$. If $X \notin \mathcal{I}$ the $\sigma$-ideal is called proper. The classical examples of proper $\sigma$-ideals are:

- the family of countable subsets of any uncountable set;

- the family of the first Baire category subsets of a topological space of the second category;

- the family of sets of measure zero with respect to a non-zero complete measure;

- the family of null subsets of a locally compact group.

A $\sigma$-ideal $\mathcal{I}$ in a group $G$ is said to be invariant if $x-A \in \mathcal{I}$ whenever $A \in \mathcal{I}$ and $x \in G$. Clearly, the first category subsets of a topological group as well as the null subsets of a locally compact Abelian group constitute invariant $\sigma$-ideals.

In the proofs of the results of 14 some abstract versions of the Fubini theorem are helpful. They can be postulated by imposing the following notions of conjugation of the considered $\sigma$-ideals. Let $\mathcal{I}_{1}$ and $\mathcal{I}_{2}$ be $\sigma$-ideals in $X$ and $X \times X$, respectively. We require that $\mathcal{I}_{1}$-a.a. appropriate sections of every set from $\mathcal{I}_{2}$ are in $\mathcal{I}_{1}$. Namely, $\mathcal{I}_{1}$ and $\mathcal{I}_{2}$ are said to be:

- conjugate if for every $A \in \mathcal{I}_{2}$ the vertical sections $\{y \in X:(x, y) \in A\}$ are in $\mathcal{I}_{1}$ for $\mathcal{I}_{1}$-a.a. $x \in X$. 
If $X$ is an Abelian group, $\mathcal{I}_{1}$ and $\mathcal{I}_{2}$ are called (see [14]):

- radially conjugate if the $\mathcal{I}_{1}$-a.a. sections $\{y \in X:(x+n y, y) \in A\}$ of any $A \in \mathcal{I}_{2}$ are in $\mathcal{I}_{1}$ for every $n \in \mathbb{Z}$,

- diagonally conjugate if they are conjugate and the $\mathcal{I}_{1}$-a.a. sections $\left\{y \in X:\left(x+2^{n} y\right.\right.$, $\left.\left.-2^{n} y\right) \in A\right\}$ and $\left\{y \in X:\left(x-2^{n} y, 2^{n} y\right) \in A\right\}$ of any $A \in \mathcal{I}_{2}$ are in $\mathcal{I}_{1}$ for every $n \in \mathbb{N} \cup\{0\}$.

Clearly, radially conjugate as well as diagonally conjugate $\sigma$-ideals are conjugate.

We need one definition more; this is a modification of that proposed by Ger in [8], [9]. For any $\sigma$-ideal $\mathcal{I}$ of subsets of a set $X$ let $\Omega(\mathcal{I})$ be the family of those $A \subset X \times X$, whose $\mathcal{I}$-a.a. vertical sections $\{y \in X:(x, y) \in A\}$ as well as horizontal sections $\{x \in X$ : $(x, y) \in A\}$ are in $\mathcal{I}$. Clearly, if $\mathcal{I}$ is an invariant proper $\sigma$-ideal in an Abelian group $G$, then $\Omega(\mathcal{I})$ is an invariant proper $\sigma$-ideal in $G \times G$, conjugate with $\mathcal{I}$.

Let $G$ be an Abelian group, $\mathcal{I}$ a proper $\sigma$-ideal in $G \times G$ and let $A$ be a subset of $G$. A function $f: A \rightarrow \mathbb{R}$ is called $\mathcal{I}$-almost convex if inequality 0.1 holds for $\mathcal{I}$-a.a. $(x, h) \in G \times G$ with $x, x+h, x-h \in A$. In [22, Th. 17.8.2] Kuczma proved what follows.

Let $\mathcal{I}_{1}$ and $\mathcal{I}_{2}$ be conjugate proper invariant $\sigma$-ideals in $\mathbb{R}^{n}$ and $\mathbb{R}^{2 n}$, respectively, fulfilling the conditions

$\left(\mathrm{K}_{1}\right)$ if $A \in \mathcal{I}_{1}$ then $a A \in \mathcal{I}_{1}$ for every $a \in \mathbb{R}$,

$\left(\mathrm{K}_{2}\right)$ if $A \in \mathcal{I}_{2}$ then $\phi^{-1}(A) \in \mathcal{I}_{2}$.

If $D \subset \mathbb{R}^{n}$ is a convex open set and $f: D \rightarrow \mathbb{R}$ is an $\mathcal{I}_{2}$-almost convex function, then there exists a unique convex function $g: D \rightarrow \mathbb{R}$ such that $g(x)=f(x)$ for $\mathcal{I}_{1}-a . a . x \in D$.

In [14], to extend Kuczma's theorem to an abstract group setting, the following general result has been proved. The condition $\left(\mathrm{Q}_{1}\right)$ below is a natural extension of $\left(\mathrm{P}_{1}\right)$ considered in Subsection 3.1, whereas the condition $\left(\mathrm{Q}_{2}\right)$ is a slight but essential modification of condition $\left(\mathrm{P}_{2}\right)$.

TheOrem 3.2 ([14, Th. 1]). Let $G$ be an Abelian group and let $\mathcal{I}_{1}$ and $\mathcal{I}_{2}$ be invariant $\sigma$-ideals in $G$ and $G \times G$, respectively, radially or diagonally conjugate and fulfilling the conditions

(Q1) if $\frac{1}{2} A \in \mathcal{I}_{1}$ then $A \in \mathcal{I}_{1}$,

$\left(\mathrm{Q}_{2}\right)$ if $A, B \subset G, A, B \notin \mathcal{I}_{1}, M \in \Omega\left(\mathcal{I}_{1}\right)$ and $N \in \mathcal{I}_{2}$, then

$$
\left[\left(\frac{1}{2} A\right) \times\left(\frac{1}{2} B\right)\right] \backslash\left[\phi^{-1}(M) \cup N\right] \neq \emptyset .
$$

If $D \subset G$ is a nonempty convex set, $(D-x) \cap(x-D) \notin \mathcal{I}_{1}$ for every $x \in D$, and $f: D \rightarrow \mathbb{R}$ is an $\mathcal{I}_{2}$-almost convex function, then there exists a convex function $g: D \rightarrow \mathbb{R}$ such that $g(x)=f(x)$ for $\mathcal{I}_{1}-a . a . x \in D$.

It can be easily checked that any conjugate proper invariant $\sigma$-ideals in $\mathbb{R}^{n}$ and $\mathbb{R}^{2 n}$, respectively, fulfilling conditions $\left(\mathrm{K}_{1}\right)$ and $\left(\mathrm{K}_{2}\right)$, are diagonally conjugate. Moreover, observe that condition $\left(K_{1}\right)$ implies $\left(Q_{1}\right)$, and $\left(Q_{1}\right),\left(K_{2}\right)$ force $\left(Q_{2}\right)$. Since $\mathcal{I}_{1}$ is invariant and proper, it follows that empty set is the unique open set belonging to $\mathcal{I}_{1}$. Thus $(D-x) \cap(x-D) \notin \mathcal{I}_{1}$ for every nonempty open set $D \subset \mathbb{R}^{n}$ and $x \in D$. Consequently, Kuczma's theorem follows directly from Theorem 3.2 and the next uniqueness result. 
Observe that the assumptions imposed on the domain $D$ of $f$ in Theorems 3.2 and 3.3 are more general than those in original Kuczma's theorem.

Theorem 3.3 ([14, Th. 2]). Let $G$ be an Abelian group and let $\mathcal{I}_{1}$ be an invariant $\sigma$-ideal in $G$ fulfilling condition $\left(\mathrm{Q}_{1}\right)$. If $D \subset G$ is a nonempty convex set, $(D-x) \cap(x-D) \notin \mathcal{I}_{1}$ for every $x \in D$ and $f_{1}, f_{2}: D \rightarrow \mathbb{R}$ are convex functions such that $f_{1}(x)=f_{2}(x)$ for $\mathcal{I}_{1}$-a.a. $x \in D$, then $f_{1}=f_{2}$.

3.3. Measure-theoretical and topological cases. There are two crucial realizations of Theorems 3.2 and 3.3 . Let us start with the first one.

Theorem 3.4 ([14, Th. 3]). Let $G$ be a locally compact Abelian group divisible by 2, with Haar measure $\lambda$, and let $D \subset G$ be a nonempty convex set such that $(D-x) \cap(x-D)$ is a null set for no $x \in D$. If $f: D \rightarrow \mathbb{R}$ and inequality (0.1) holds for $\lambda^{2}$-a.a. $(x, h) \in G \times G$ with $x, x+h, x-h \in D$, then there exists a unique convex function $g: D \rightarrow \mathbb{R}$ such that $g(x)=f(x)$ for $\lambda$-a.a. $x \in D$.

Theorem 3.4 can be derived from Theorems 3.2 and 3.3 due to a series of lemmas proved in [14. They show that the divisibility of $G$ by 2 forces conditions $\left(\mathrm{Q}_{1}\right)$ and $\left(\mathrm{Q}_{2}\right)$ with the $\sigma$-ideals $\mathcal{I}_{1}$ and $\mathcal{I}_{2}$ of null sets in $G$ and $G \times G$, respectively. On the other hand, Theorem 3.4 generalizes Theorem 3.1 where $f$ is assumed to be defined on the whole group.

For a topological version of Theorems 3.2 and 3.3 let $G$ be an Abelian topological group with a countable base. Then, according to the Kuratowski-Ulam theorem (cf. [28, Chap. 15]), the first category subsets of $G$ and $G \times G$, respectively, form invariant radially conjugate $\sigma$-ideals.

Theorem 3.5 ([14, Th. 4]). Let $G$ be a locally compact Abelian group with a countable base. Assume that $G$ is divisible by 2 and has only finitely many elements of order 2 . Let $D \subset G$ be a nonempty convex set such that $(D-x) \cap(x-D)$ is of the second category for every $x \in D$. If $f: D \rightarrow \mathbb{R}$ and there is a first category subset $P$ of $G \times G$ such that inequality (0.1) holds for every $(x, h) \in(G \times G) \backslash P$ with $x, x+h, x-h \in D$, then there exist a first category subset $Q$ of $G$ and a unique convex function $g: D \rightarrow \mathbb{R}$ such that $g(x)=f(x)$ for every $x \in D \backslash Q$.

The $n$-dimensional torus serves as an example of a non-trivial, that is not uniquely divided by 2 , group satisfying the assumptions of Theorem 3.5 .

3.4. Some consequences. We complete this section with two consequences of some results presented here. The first one states that, under appropriate conditions imposed on the group $G$, every measurable function satisfying a condition related to almost convexity is equal to a continuous convex function almost everywhere.

TheOREM 3.6 ([14, Th. 5]). Let $G$ be a $\sigma$-compact locally compact Abelian group, divisible by 2 , with Haar measure $\lambda$, and let $D \subset G$ be a nonempty convex open set. Let $f: D \rightarrow \mathbb{R}$ be a measurable function. Assume that there is a dense subset $B$ of $G$ such that inequality (0.1) holds for all $x \in G$ and $h \in B$ with $x, x+h, x-h \in D$. Then there exists a continuous convex function $g: D \rightarrow \mathbb{R}$ such that $g(x)=f(x) \lambda$-a.e. in $D$. 
The main tools used in the proof of the above result are Theorems 3.4 and 2.9 . Making use of Theorems 3.5 and 2.10 one can deduce the following topological version of Theorem 3.6 .

THEOREM 3.7 ([14, Th. 6]). Let $G$ be a locally compact Abelian group with a countable base. Assume that $G$ is divisible by 2 and has only finitely many elements of order 2 . Let $D \subset G$ be a nonempty convex open set and let $f: D \rightarrow \mathbb{R}$ be a function with the Baire property. Assume that there is a dense subset $B$ of $G$ such that inequality (0.1) holds for every $x \in G$ and $h \in B$ with $x, x+h, x-h \in D$. Then there exist a continuous convex function $g: D \rightarrow \mathbb{R}$ and a first category subset $Q$ of $G$ such that $g(x)=f(x)$ for every $x \in G \backslash Q$.

\subsection{Problems}

1. Answer the question whether or not Theorem 3.1 is valid in every locally compact Abelian group.

2. Is it possible to relax or even cancel the divisibility assumption imposed on $G$ in Theorem 3.5? Examine the assertion of this result in the Cartesian product of infinitely but countably many copies of the circle $S^{1}$.

3. Check if the local compactness of the group $G$ is essential for the validity of Theorem 3.5 .

4. Prove or disprove: Theorem 3.6 is true in every locally compact Abelian group.

5. In the paper 35 , replacing inequality 0.2 by the inclusion

$$
\frac{F(x)+F(y)}{2} \subset F\left(\frac{x+y}{2}\right)
$$

Sadowska studied almost convex set-valued functions defined on a convex open subset of a linear topological space. Examine that notion and Sadowska's result in a group setting, for some individual $\sigma$-ideals or abstract $\sigma$-ideals.

6 . Versions of Kuczma's theorem for almost $t$-convex and almost Wright-convex functions, defined on a convex subset of a linear space over the reals, are known due to Adamek [1. Generalize his results to groups.

4. Applications. We confine ourselves to two topics, touching only on possible applications.

4.1. Improving regularity of solutions of a functional equation. It turns out that Kuczma's theorem on almost convex functions (see [21] and [22, Th. 17.8.2]; cf. also Section 3.1 of the present paper) can be useful while proving that Lebesgue measurable solutions of some functional equations in a single variable are, in fact, continuous. A pertinent exemplification of this is the following result.

THEOREM 4.1 ([13, Prop. 3.3.(ii)]). Let $p_{1}, \ldots, p_{k}$ be positive numbers and let $a_{1}, \ldots, a_{k}$ be non-zero reals generating a dense subgroup of $\mathbb{R}$. If $f: \mathbb{R} \rightarrow \mathbb{R}$ is a non-negative measurable function satisfying the equality

$$
f(x)=\sum_{i=1}^{k} p_{i} f\left(x+a_{i}\right)
$$


a.e. in $\mathbb{R}$, then either $f(x)=0$ for a.a. $x \in \mathbb{R}$, or there is a positive continuous solution $g: \mathbb{R} \rightarrow \mathbb{R}$ of equation (4.1) such that $f(x)=g(x)$ for a.e. $x \in \mathbb{R}$; in addition, $g$ is logarithmically convex:

$$
g\left(\frac{x+y}{2}\right) \leq \sqrt{g(x) g(y)}, \quad x, y \in \mathbb{R} .
$$

This is one of the main steps in determining all the non-negative measurable solutions of 4.1] (see [13, Th. 3.1], also [23] for another argument). As the reference [13] is hardly obtainable, below we give a sketch of the proof of a special variant of Theorem 4.1 to see the crucial role of Kuczma's theorem while proving Theorem 4.1. The condition $f(0+)=0$ is not essential for the validity of Theorem 4.2 (cf. [13, Lemma 1.3]). However, imposing this assumption we can give a simpler argument.

THEOREM 4.2. Let $a, b$ be positive numbers such that $\log a / \log b$ is irrational. If $f$ : $(0, \infty) \rightarrow \mathbb{R}$ is a non-negative measurable function such that $f(0+)=0$ and the equality

$$
f(x)=f(a x)+f(b x)
$$

holds a.e. in $(0, \infty)$, then either $f(x)=0$ for a.a. $x \in(0, \infty)$, or there is a positive continuous solution $g:(0, \infty) \rightarrow \mathbb{R}$ of equation 4.2 such that $f(x)=g(x)$ for a.a. $x \in(0, \infty)$; in addition, $g$ is geometrically convex:

$$
g(\sqrt{x y}) \leq \sqrt{g(x) g(y)}, \quad x, y \in(0, \infty) .
$$

Proof. Let $E \subset(0, \infty)$ be a null set such that equality 4.2 holds for every $x \in(0, \infty) \backslash E$. Then the set

$$
E_{0}=\bigcup_{i=-\infty}^{\infty} \bigcup_{j=-\infty}^{\infty} a^{i} b^{j} E
$$

is of measure zero. Moreover, $a E_{0}=b E_{0}=E_{0}$. Thus, replacing $f$ by the function $f_{0}:(0, \infty) \rightarrow \mathbb{R}$ given by

$$
f_{0}(x)= \begin{cases}f(x), & \text { if } x \in(0, \infty) \backslash E_{0}, \\ 0, & \text { if } x \in E_{0},\end{cases}
$$

we may assume that $f$ satisfies 4.2 for every $x \in(0, \infty)$.

Modifying slightly the proof of [17. Lemma 2] we deduce that actually $a, b \in(0,1)$. It can be easily checked that the argument used in [17, Lemma 6] remains valid if the assumption " $f \in \mathcal{F}$ " is replaced by " $f$ is non-negative and $f(0+)=0$ " there. Making use of this fact we infer that

$$
f(x)^{2} \leq f\left(a^{i} b^{j} x\right) f\left(a^{-i} b^{-j} x\right), \quad x \in(0, \infty), \quad i, j \in \mathbb{Z} .
$$

Thus, putting $\varphi=f \circ \exp$, we come to a non-negative measurable function $\varphi: \mathbb{R} \rightarrow \mathbb{R}$ satisfying the condition

$$
\varphi(x)^{2} \leq \varphi(x+i \log a+j \log b) \varphi(x-i \log a-j \log b), \quad x \in \mathbb{R}, \quad i, j \in \mathbb{Z} .
$$

In other words,

$$
\varphi(x)^{2} \leq \varphi(x+h) \varphi(x-h), \quad x \in \mathbb{R}, \quad h \in B,
$$

where $B=\mathbb{Z} \log a+\mathbb{Z} \log b$. Since the number $\log a / \log b$ is irrational, $B$ is a dense subset of $\mathbb{R}$. 
The set $T=\left\{(x, h) \in \mathbb{R}^{2}: \varphi(x)^{2}>\varphi(x+h) \varphi(x-h)\right\}$ is measurable. Fix a real $h$ and a sequence $\left(h_{n}\right)_{n \in \mathbb{N}}$ of elements of $B$ tending to $h$. By [23, Lemma 2] there is a subsequence $\left(h_{n_{m}}\right)_{m \in \mathbb{N}}$ of $\left(h_{n}\right)_{n \in \mathbb{N}}$ and a null set $E(h) \subset \mathbb{R}$ such that

$$
\varphi\left(x+h_{n_{m}}\right) \rightarrow \varphi(x+h) \quad \text { and } \quad \varphi\left(x-h_{n_{m}}\right) \rightarrow \varphi(x-h), \quad x \in \mathbb{R} \backslash E(h) .
$$

Then

$$
\varphi(x)^{2} \leq \varphi\left(x+h_{n_{m}}\right) \varphi\left(x-h_{n_{m}}\right), \quad x \in \mathbb{R} \backslash E(h), \quad m \in \mathbb{N},
$$

whence

$$
\varphi(x)^{2} \leq \varphi(x+h) \varphi(x-h), \quad x \in \mathbb{R} \backslash E(h) .
$$

Thus $\left\{(x, h) \in \mathbb{R}^{2}: \varphi(x)^{2}>\varphi(x+h) \varphi(x-h)\right\} \subset E(h)$, and, consequently, the $h$-section of $T$ is a null set for every $h \in \mathbb{R}$. By Fubini's theorem this means that $T$ is a null set.

If the set $Z=\{x \in \mathbb{R}: \varphi(x)=0\}$ is of full measure, then $\varphi(x)=0$ for a.a. $x \in \mathbb{R}$, that is $f(x)=0$ for a.a. $x \in(0, \infty)$. So assume that $|\mathbb{R} \backslash Z|>0$. Since $|T|=0$, there is a null set $N \subset \mathbb{R}$ such that the $x$-section of $T$ is a null set for every $x \in \mathbb{R} \backslash N$. Fix a point $x_{0} \in \mathbb{R} \backslash(N \cup Z)$. Then $\varphi\left(x_{0}\right)>0$ and $\left|\left\{y \in \mathbb{R}:\left(x_{0}, y\right) \in T\right\}\right|=0$ which means that

$$
0<\varphi\left(x_{0}\right)^{2} \leq \varphi\left(x_{0}+h\right) \varphi\left(x_{0}-h\right) \quad \text { for a.a. } h \in \mathbb{R} .
$$

Thus $\varphi\left(x_{0}+h\right)>0$ for a.a. $h \in \mathbb{R}$, i.e. the set $Z$ has measure zero. Putting

$$
F(x)= \begin{cases}\log \varphi(x), & \text { if } x \in \mathbb{R} \backslash Z, \\ 0, & \text { if } x \in Z,\end{cases}
$$

we come to a measurable function $F: \mathbb{R} \rightarrow \mathbb{R}$ which, satisfying the condition

$$
2 F(x) \leq F(x+h)+F(x-h), \quad(x, h) \in \mathbb{R}^{2} \backslash T,
$$

is almost convex. By Kuczma's theorem there exists a convex function $G: \mathbb{R} \rightarrow \mathbb{R}$ such that $F(x)=G(x)$ for a.a. $x \in \mathbb{R}$. Since $F$ is measurable and $G$ is convex, the last one is continuous. Consequently, the function $g=\exp \circ G \circ \log$ is positive continuous geometrically convex. Moreover, $f(x)=g(x)$ for a.a. $x \in(0, \infty)$. In particular, $g$ being continuous satisfies equation 4.2 everywhere in $(0, \infty)$.

At the very end of the above proof one can make use of Theorem 3.6 instead of Kuczma's theorem.

Theorem 4.1 was extended to functions defined in $\mathbb{R}^{n}$ and measurable with respect to some $\sigma$-fields containing Borel subsets by Grinč [10, Theorem]. In particular, a topological version of it is proved there (see [10, Cor. 1]). The main tool of both proofs is Kuczma's theorem [22, Th. 17.8.2]. However, while proving [10, Cor. 1] one can use also Theorem 3.7.

Theorem 4.1 and [10, Theorem] make up the first step in determining the form of all non-negative solutions of equation [4.1], Lebesgue measurable [13. Th. 3.1] and with the Baire property [10, Cor. 2]. All the Lebesgue measurable non-negative solutions of (4.1) were found yet in [23] by Laczkovich, by using quite different argument. Equation 4.2 was studied in the paper [17], where all the continuous solutions $f:(0, \infty) \rightarrow \mathbb{R}$ fulfilling $f(0+)=0$ and not vanishing in a vicinity of 0 have been determined (see [17, Th. 1]). The form of all of them allows to determine all weak generalized stable distributions (see [17, Th. 4]). 
4.2. Integer programming. Integer programming is important in economics due to its usefulness in solving optimization problems and problems arising in operation research and management science (cf., for instance, the classical optimal fleet mix problem). This is a wide topic, studied for many years and having a vast literature. Here we would like only to point out some close relations between integer programming and convex functions defined on subsets of a very special group, viz. the group $\mathbb{Z}$ of all integers.

One of the most fundamental models in the discrete optimization is that of matroids which can be viewed as some discrete convex, in a sense, structures. This motivates the quest for a general theory of discrete convexity. There are various approachs to that notion and many, in the main not equivalent, definitions of it. The reader is referred to the article [7] by Favati and Tardella, the book [25] by Murota, also the papers [38] due to Yüceer, 26] by Murota and Shioura, and [5] by Cambini, Riccardi and Yüceer. The last paper yields also a new concept of discrete convex function defined on a suitable subset of $\mathbb{Z}^{n}$; the inequality describing this notion is equivalent to inequality 0.1 . In the paper 4] Cambini and Riccardi have been studied the case $n=1$ more thoroughly and compared discrete convexity in that situation with some discrete quasiconvexity concepts.

Clearly one of the most essential problem of optimization is discrete convex function minimization. However, determining minima of strictly convex functions (i.e. such convex function that inequality $(0.1)$ is sharp whenever $h \neq 0$ ) one can realize that, in contrast to the classical theory of convexity, discrete convex functions admit more than one global minimum. The function $f: \mathbb{Z} \rightarrow \mathbb{R}$, given by $f(x)=\left|x-\frac{1}{2}\right|-\frac{1}{2}$, serves as an example: it is strictly convex and takes the global minimum 0 at 0 and 1 . The paper [4] provides also two efficient algorithms for the determining the minimum points of dicrete strictly convex functions defined on "intervals" of integers.

For some different ideas of discrete convexity, essential in economics and other areas computer aided, the reader is referred to the paper [37] by Tabor\&Tabor and Misztal. There convexity is considered both for finite sets and functions with such domains.

Acknowledgements. I am indebted to the referee for calling my attention to reference [29].

\section{References}

[1] M. Adamek, Almost $\lambda$-convex and almost Wright-convex functions, Math. Slovaca 53 (2003), 67-73.

[2] F. Bernstein, G. Doetsch, Zur Theorie der konvexen Funktionen, Math. Ann. 76 (1915), $514-526$.

[3] H. Blumberg, On convex functions, Trans. Amer. Math. Soc. 29 (1919), 40-44.

[4] R. Cambini, R. Riccardi, On discrete quasiconvexity concepts for single variable scalar functions, Taiwanese J. Math. 13 (2009), 837-853.

[5] R. Cambini, R. Riccardi, Ü. Yüceer, An approach to discrete convexity and its use in an optimal fleet mix problem, in: Generalized Convexity and Related Topics, Lecture Notes in Econom. and Math. Systems 583, Springer, Berlin, 2007, 133-148.

[6] A. Chademan, F. Mirzapour, Midconvex functions in locally compact groups, Proc. Amer. Math. Soc. 127 (1999), 2961-2968. 
[7] P. Favati, F. Tardella, Convexity in nonlinear integer programming, Ricerca Operativa 53 (1990), 3-44.

[8] R. Ger, On some functional equations with a restricted domain, Fund. Math. 89 (1975), 131-149.

[9] R. Ger, On some functional equations with a restricted domain. II, Fund. Math. 98 (1978), 250-272.

[10] M. Grinč, On non-negative measurable solutions of a difference functional equation, Positivity 2 (1998), 221-228.

[11] E. Hewitt, K. A. Ross, Abstract Harmonic Analysis. I, Grundlehren Math. Wiss. 115, Springer, Berlin, 1979.

[12] E. Hopf, Über die Zusammenhänge zwischen gewissen höheren Differenzenquotienten reeller Funktionen einer reellen Variablen und deren Differenzierbarkeitseigenschaften, Dissertation, Universität Berlin, 1926.

[13] W. Jarczyk, A recurrent method of solving iterative functional equations, Prace Nauk. Uniw. Slask. Katowic. 1206, Uniwersytet Śląski, Katowice, 1991.

[14] W. Jarczyk, Almost convexity on Abelian groups, Aequationes Math. 80 (2010), 141-154.

[15] W. Jarczyk, M. Laczkovich, Convexity on Abelian groups, J. Convex Anal. 16 (2009), $33-48$.

[16] W. Jarczyk, M. Laczkovich, Almost convex functions on locally compact Abelian groups, Math. Inequal. Appl. 13 (2010), 217-225.

[17] W. Jarczyk, J. K. Misiewicz, On weak generalized stability and $(c, d)$-pseudostable random variables via functional equations, J. Theoret. Probab. 22 (2009), 482-505.

[18] J. L. W. V. Jensen, Sur les fonctions convexes et les inégalités entre les valeurs moyennes, Acta Math. 30 (1906), 175-193.

[19] M. R. Kaufman, Extension of functionals and inequalities on an Abelian semi-group, Proc. Amer. Math. Soc. 17 (1966), 83-85.

[20] M. R. Kaufman, Interpolation of additive functionals, Studia Math. 27 (1966), 269-272.

[21] M. Kuczma, Almost convex functions, Colloq. Math. 21 (1970), 279-284.

[22] M. Kuczma, An Introduction to the Theory of Functional Equations and Inequalities. Cauchy's Equation and Jensen's Inequality, Birkhäuser, Basel, 2009.

[23] M. Laczkovich, Nonnegative measurable solutions of difference equations, J. London Math. Soc. (2) 34 (1986), 139-147.

[24] M. R. Mehdi, On convex functions, J. London Math. Soc. 39 (1964), 321-326.

[25] K. Murota, Discrete Convex Analysis, SIAM Monogr. Discrete Math. Appl., SIAM, Philadelphia, PA, 2003.

[26] K. Murota, A. Shioura, Quasi M-convex and L-convex functions: quasiconvexity in discrete optimization, Discrete Appl. Math. 131 (2003), 467-494.

[27] A. Ostrowski, Über die Funktionalgleichung der Exponentialfunktion und verwandte Funktionalgleichungen, Jahresber. Deutsch. Math.-Verein. 38 (1929), 54-62.

[28] J. C. Oxtoby, Measure and Category, Grad. Texts in Math. 2, Springer, New York, 1971.

[29] A. Pinkus, D. Wulbert, Extending n-convex functions, Studia Math. 171 (2005), 125-152. 
[30] T. Popoviciu, Sur quelques propriétés des fonctions d'une ou de deux variables réelles, thèse, Faculté des Sciences de Paris, 1933.

[31] T. Popoviciu, Les Fonctions Convexes, Actualités Sci. Ind. 992, Hermann et Cie, Paris, 1944.

[32] A. W. Roberts, D. E. Varberg, Convex Functions, Pure and Applied Mathematics 57, Academic Press, New York, 1973.

[33] G. Rodé, Eine abstrakte Version des Satzes von Hahn-Banach, Arch. Math. (Basel) 31 (1978), 474-481.

[34] J. J. Rotman, An Introduction to the Theory of Groups, Grad. Texts in Math. 148, Springer, New York, 1995.

[35] E. Sadowska, Almost midconvex and almost convex set-valued functions, Math. Slovaca 50 (2000), 453-461.

[36] W. Sierpiński, Sur les fonctions convexes mesurables, Fund. Math. 1 (1920), 125-128.

[37] J. Tabor, J. Tabor, K. Misztal, Midconvexity for finite sets, manuscript.

[38] Ü. Yüceer, Discrete convexity: convexity for functions defined on discrete spaces, Discrete Appl. Math. 119 (2002), 297-304. 